\title{
The Study on Community Health Education of Empty Nest Elderly
}

\author{
Ling Gao ${ }^{1}$, Xue Cao ${ }^{1,2}$, Min Zhang ${ }^{1}$ \\ ${ }^{1}$ Beihua University, Jilin, China \\ ${ }^{2}$ Baicheng Medical College, Baicheng, China \\ Email: bhgaoling@sina.cn
}

Received April 2013

\begin{abstract}
Objective: To study the demands of community health education on the empty nest elderly and analyse the related factors. Methods: The 36-Item Short-Form Health Survey (SF-36), activities of daily living (ADL) and the community health services satisfaction scale were used to investigate the community health service demand and supply of 358 empty nesters. Results: The results showed that the ability of self-care was worse and needed more nursing or caring from others in the empty nest elderly; the empty nest elderly, in comparison with the non-empty-nest elderly, had lower physical and mental scores. With the age changing, the requirements of community health services were different in empty nest elderly and the satisfaction of community health service was low in the empty nest (39.1\%). Conclusions: Empty nest elderly generally needed the different degree of community health education demands. The utilization rates of community health education were low.
\end{abstract}

Keywords: Empty Nest Elderly; Community Health Education

\section{Introduction}

With the accelerating pace of population aging population and the miniaturization trend of the family structure, the proportion of empty nesters will further improve [1]. In China, the number of empty-nest families is on the rise, especially in some towns. Empty nests are a special group in society. As the nesters are unaccompanied and unattended, it usually causes many problems in physical, psychological and social field.

Community health service takes health as the center, the family as a unit and community-wide, which is the health service system of prevention, care, rehabilitation, health care and health education [2]. The elderly, children and the disabled are the main service targets of community health service. With the aging of population, rapid development of empty nests, nesters have gradually become an object group of community health service. The characteristics of empty nesters are of large quantity, there will be more service projects and difficulties. Thus they will become the work focus of the community health service. Community health education is the core role in the community health service, which could guide the elders (to treat and prevent diseases) and improve the quality of life. Compared with some developed countries, the development of Chinese community health education is relatively late and the system is still not yet perfect.

In this study, the community health education demand and supply of empty nesters were used to study to constantly improve the community health service, which provided the scientific base for the government and relevant departments, so as to establish a perfect community health services for the aged system and further improved the life quality of the empty nesters.

\section{Methods}

\subsection{Subjects}

A total of 801 empty nesters in Jilin County were invited to participate in the study, with informed consent after receiving information about the goal and the method of the investigation, together with approval from the local government authorities, 728 empty nesters completed the questionnaire thoroughly. 45 elders were not able to complete the questionnaire because of physical or mental disabilities, and 28 elders returned incomplete data. The response rate of effective questionnaires was $90.88 \%$ (728/801). In the study, elders were classified as empty nest group and non-empty-nest group by distinguishing, "Are you living with any of your children together?" If the answer was "no" instead of "yes", this was seen as an empty nester [2].

\subsection{Instruments}

The 36-Item Short-Form Health Survey (SF-36), devel- 
oped in the US and employed in population surveys around the world, has been shown to be a valid measure of general health in population studies. Activities of daily living (ADL) is composed with independent living table (physiscal self-maintenance scale, PSMS) and instrumental actiities of daily liing scale (instrumental activities of daily living scale, IADL), it is used to evaluate the ability of daily life [3].

\subsection{Data Analysis}

The association between SF-36 scores in both the empty nest and non-empty-nest elderly were explored with Pearson's correlations. We used the linear regression model with conditional stepwise analysis to study the significant factors predictive of loneliness. All data were analyzed with the SPSS 13.0 statistical analysis software package [3].

\section{Results}

\subsection{General Information}

The resulting data included 358 (49.2\%) empty nest elder adults and 370 (50.8\%) non-empty-nest elder adults. Ages of these respondents ranged from 60 to 81 years (mean $=61.7 \pm 6.78$ years) for the empty-nest group and from 60 to 83 years (mean $=63.1 \pm 7.21$ years) for the non-empty-nest group. Most of the empty nest and nonempty-nest elderly had finished their education to the primary school level, and had been in the medical system. There were no significant differences between the empty nest and non-empty-nest group in gender, age, educational level, marital status and insurance coverage ( $\mathrm{P}>$ 0.05) (Table 1). The empty nest elderly, in comparison with the non-empty-nest elderly, had lower physical (95\% $\mathrm{CI}=0.217$ to $6.131, \mathrm{t}=2.208, \mathrm{df}=533)$ and mental $(95 \%$ $\mathrm{CI}=0.812$ to $6.281, \mathrm{t}=2.511, \mathrm{df}=523.7$ ) scores from the SF-36. 581 elders were suffered from chronic diseases (79.8\%), 357 elders were suffered from one disease (49\%), 114 elders had two kinds of diseases (19.9\%), 22 elders had three kinds of diseases (15.7\%) and 147 elders without evidence of disease (20.2\%). The chronic diseases threatened the elderly health are followed by hypertension $(47.3 \%)$, diabetes $(24.8 \%)$, osteoarthritis (10.8\%), stroke (9.1\%) and heart disease (8\%).

\subsection{The Ability of Daily Life Were Decreased}

Activities of daily living (ADL) is composed with independent living skills (physical self-maintenance scale, PSMS, including food, clothing, wash, and leaving the toilet, walk and a bath ) and instrumental daily living skills (IADL, including making phone calls, shopping, cooking, housework, laundry, using the traffic tools, medication and providing for oneself economy). The above
Table 1. The comparison of community health services between empty nest and not empty nest elderly.

\begin{tabular}{|c|c|c|c|c|c|}
\hline & \multicolumn{2}{|c|}{$\begin{array}{c}\text { Empty nest group } \\
(\mathrm{n}=358)\end{array}$} & \multicolumn{2}{|c|}{$\begin{array}{l}\text { Not Empty nest } \\
\text { group }(n=370)\end{array}$} & \multirow[b]{2}{*}{$\mathrm{p}$} \\
\hline & $\mathrm{n}$ & $\%$ & $\mathrm{n}$ & $\%$ & \\
\hline Health examination & 248 & 69.2 & 256 & 69.2 & $>0.05$ \\
\hline $\begin{array}{l}\text { Door medical } \\
\text { services }\end{array}$ & 212 & 59.1 & 170 & 48.1 & $<0.05$ \\
\hline Health guide & 218 & 60.9 & 221 & 59.7 & $>0.05$ \\
\hline Domestic sickbed & 96 & 26.8 & 57 & 15.4 & $<0.05$ \\
\hline Rehabilitation care & 68 & 19 & 71 & 19.1 & $>0.05$ \\
\hline Other need & 21 & 5.8 & 27 & 6.4 & $>0.05$ \\
\hline No need & 35 & 9.8 & 36 & 9.7 & $>0.05$ \\
\hline
\end{tabular}

14 items daily life events were used in this study. "Do not help, some difficulties to need help and completely cannot provide for oneself" were used to evaluated the capability. The results showed that the ADL of elders drop as the growth of the age, the ability of self-care were worse. In comparison with the non-empty-nest, the empty nest elderly needed more nursing or caring from others.

\subsection{The Comparison of Community Health Education Demands in Different Age of the Empty Nest Elderly}

The community health service demands were studied in different age of the empty nest elderly. The results showed that the 80 years old group urgently needed door medical services (49.5\%), compared with other services, the differences were statistically significant $(\mathrm{P}<0.05)$; compared with other services, the demand of rehabilitation health care was highest in 70 years old group (42.1\%); the demand of health guide was highest in 60 years old group (42\%) (Table 2); With the age changed, the requirements of community health services were different.

\section{Discussion}

The sick and aging made it impossible to provide for themselves which made the empty nest elderly need more help. Diagnosis and treatment of domestic sickbed demands were high in empty nest elderly. This phenomenon hints that the community health education service development space is very large [4]. Compared with the non-empty-nest elderly, the empty nest elderly had poor psychological condition. Therefore, the community health service should pay attention to the empty nest elderly on health services.

The results showed that the demands of the community health service had differences among different age of the empty nest elderly. Group of 80 -year-old urgently needed door health services (49.5\%). This might be rela- 
Table 2. The comparison of community health education services between different age empty nest elderly.

\begin{tabular}{cccc}
\hline & $\begin{array}{c}60 \text { years old } \\
\text { group } \\
(\mathrm{n}=138 \%)\end{array}$ & $\begin{array}{c}60 \text { years old } \\
\text { group } \\
(\mathrm{n}=138 \%)\end{array}$ & $\begin{array}{c}80 \text { years old } \\
\text { group } \\
(\mathrm{n}=99 \%)\end{array}$ \\
\hline $\begin{array}{c}\text { Health } \\
\text { examination }\end{array}$ & $33(23.9)$ & $21(17.4)$ & $10(10.1)$ \\
$\begin{array}{c}\text { Health guide } \\
\text { Door medical } \\
\text { services }\end{array}$ & $58(42)^{*}$ & $20(16.5)$ & $9(9.1)$ \\
$\begin{array}{c}\text { Domestic sickbed } \\
\text { Rehabilitation care }\end{array}$ & $8(5(14)$ & $11(9.1)$ & $49(49.5)^{* *}$ \\
Other need & $4(2.9)$ & $10(8.3)$ & $19(19.2)$ \\
No need & $4(2.9)$ & $51(42.1)^{\#}$ & $10(10.1)$ \\
\hline
\end{tabular}

*denotes a significant difference to other services $(\mathrm{P}<0.05)$; "denotes a significant difference to other services $(\mathrm{P}<0.05) ;{ }^{* *}$ denotes a significant difference to other services $(\mathrm{P}<0.05)$.

tive to two reasons. On the one hand, as the growth of the age, the rates of chronic diseases increased; on the other hand, the children of empty nest elderly were not at nearby. They were lack of family chaperone in sick times. So the demand of door medical services is more urgent. Groups of 70 years old and 60 years old needed the demands of rehabilitation medical care and health guide. It might be relative to higher culture, a certain economic income, small economic pressure in young empty nest elderly. They could take the initiative for health knowledge and have strong health concept and self-health care. Therefore, community health service center should actively explore new mode and a new method to adapt the various demands of empty nest elderly in different age levels [5].

According to the results of this survey, the community health service center provided service projects. The demands of empty nest elderly were as follows: regular health examination (69.2\%), the door medical services (59.1\%), health guide (60.9\%) and rehabilitation health care (19\%). Only a few old men take part in the regular health education and health services. Some community did not begin domestic sickbed medical care and rehabilitation services. The supply of community health service, especially active supply had bigger difference. The main reasons are that China's community health service network is still incomplete and inadequately invested. The function is not strong and the personnel quality is not high. The impact is not strong. These reasons all hinder the development of community health services. The findings from our study could provide the government with some information about the demands of community health services in the empty nest elderly. Results of the study suggest that we need to be aware of the empty nest elder adults' demands in community health services.
According to the actual situation in China, the publicity of community health service should be strengthened through the billboards, regularly health seminars, the prevention and treatment of common diseases to raise the elderly self-care consciousness, self-health care and psychological health level. The health records were established for each empty nest elderly to take countermeasures to prevent and control measures to improve the quality of life among the empty nest elderly. Actions must be taken in the care for the elderly, especially for the empty nest elderly [6].

\section{Acknowledgements}

This work was partially supported by the Social science foundation project of Jilin province (2011B146).

\section{REFERENCES}

[1] W. Hou, H. Fan, J. Xu, F. Wang, Y. Chai, H. Xu, Y. Li, L. Liu, B. Wang, J. Jin and Z. Lu, "Service Functions of Private Community Health Stations in China: A Comparison Analysis with Government-Sponsored Community Health Stations," Journal of Huazhong University of Science and Technology [Medical Sciences], Vol. 32, No. 2, 2012, pp. 159-166.

http://dx.doi.org/10.1007/s11596-012-0029-3

[2] H. Hu, W. Liang, M. Liu, L. Li, Z. Li, T. Li, J. Wang, T. Shi, S. Han, M. Su, X. Peng, Y. Peng, W. Zhao, B. Wang, P. Zhang and W. Zhu, "Establishment and Evaluation of a Model of a Community Health Service in an Underdeveloped Area of China,” Public Health, Vol. 124, No. 4, 2010, pp. 206-217. http://dx.doi.org/10.1016/j.puhe.2010.01.015

[3] L. J. Liu and Q. Guo, "Loneliness and Health-Related Quality of Life for the Empty Nest Elderly in the Rural Area of a Mountainous County in China," Quality of Life Research, Vol. 16, No. 8, 2007, pp. 1275-1280.

http://dx.doi.org/10.1007/s11136-007-9250-0

[4] L. J. Liu and Q. Guo, "Life Satisfaction in a Sample of Empty-Nest Elderly: A Survey in the Rural Area of a Mountainous County in China," Quality of Life Research, Vol. 17, No. 6, 2008, pp. 823-830. http://dx.doi.org/10.1007/s11136-008-9370-1

[5] D. Su, X. N. Wu, Y. X. Zhang, H. P. Li, W. L. Wang, J. P. Zhang and L. S. Zhou, "Depression and Social Support between China Rural and Urban Empty-Nest Elderly,” Archives of Gerontology and Geriatrics, Vol. 55, No. 3, 2012, pp. 564-569.

http://dx.doi.org/10.1016/j.archger.2012.06.006

[6] J. Wang and X. Zhao, "Empty Nest Syndrome in China," International Journal of Social Psychiatry, Vol. 58, No. 1, 2012, p. 110. http://dx.doi.org/10.1177/0020764011418406 